\title{
Two Cases of Near-Missed Intestinal Perforation at the Initial Survey of Trauma Patients
}

\author{
Joongsuck Kim, Oh Sang Kwon, Kyoung Hwan Kim, Min Koo Lee, Ho Hyoung Lee, \\ Sung Ho Han, Seok Jin Yang \\ Department of traumatology, Jeju regional trauma center, Cheju Halla General Hospital , Jeju-si, Jeju-do, Republic of Korea
}

With the advent of computed tomography scans, trauma surgeons can easily detect intestinal perforations. However, the signs and scans remain non-definitive in some cases. Here, we present two cases of near-missed intestinal perforation: one case was clearly suspected of intestinal perforation, whereas the other was not so apparent. One should consider the possibility of occult intestinal injury for the abdominal trauma.

Key Words: Intestine; Perforation; Computed Tomography

(Trauma Image Proced 2018(2):53-55)

\section{INTRODUCTION}

Untreated intestinal perforation sustained following a blunt trauma mostly results in generalized peritonitis, ultimately leading to sepsis. Most cases warrant surgical repair. Thus, any signs and symptoms of intestinal perforation should be crucially detected, and a general surgeon should be immediately consulted. These signs include abdominal tenderness, abdominal distention, fever, and leukocytosis. With the advent of computed tomography (CT) scans, the detection rate of perforation improved. However, the signs and scans remain non-definitive in some cases. Here, we present two cases of near-missed intestinal perforation: one case was clearly suspected of intestinal perforation, whereas the other was not so apparent.

\section{First case}

A 55-year-old male presented to the emergency room (ER) after sustaining a fall from an approximately 3-m-high site with complaints of severe abdominal pain. His vital signs were stable. The CT scan revealed an apparent small pneumoperitoneum (Fig. 1). The patient was immediately brought to the operating room (OR) for exploratory laparotomy, which revealed a 2-cm-sized laceration at the antimesenteric border of the proximal jejunum. Minimal bowel content spillage was noted and there were no other internal organ injuries. The laceration was primarily repaired. The patient was discharged on postoperative day 10 without complication.

\section{Second case}

A 51-year-old male presented to the ER after being

Received: October 11, 2018 Revised: November 9, 2018 Accepted: November 13, 2018

Correspondence to: Joongsuck Kim, Department of traumatology, Jeju regional trauma center, Cheju Halla General Hospital, 65, Doryeong-ro, Jeju-si, Jeju-do, Republic of Korea

Tel: 82-064-740-5000, Fax: 82-064-743-3110, E-mail: jsknight68@hanmail.net

Copyright (c) 2018 Korean Association for Research, Procedures and Education on Trauma. All rights reserved.

@This is an open-access article distributed under the terms of the Creative Commons Attribution Non-Commercial License (http://creativecommons.org/ licenses/by-nc/4.0) which permits unrestricted noncommercial use, distribution, and reproduction in any medium, provided the original work is properly cited 


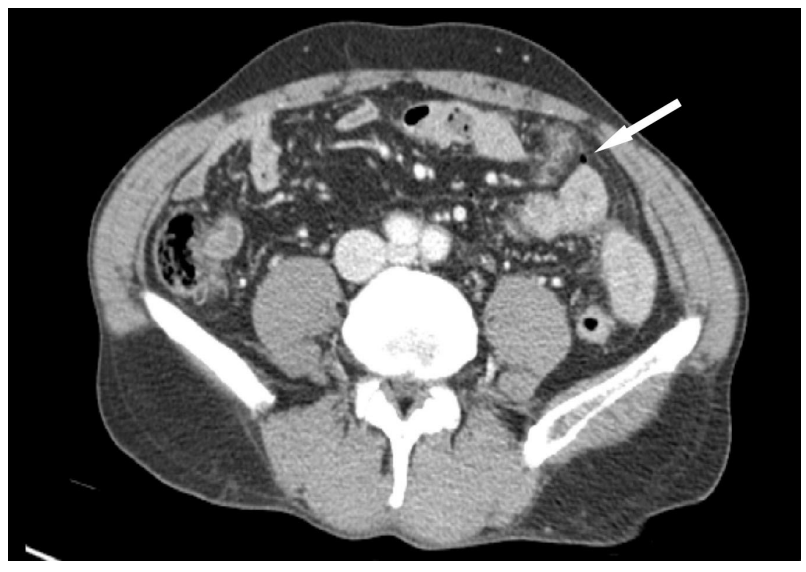

Fig. 1. Abdominal CT scan of the first patient revealing a very small amount of free air (indicated by white arrow)

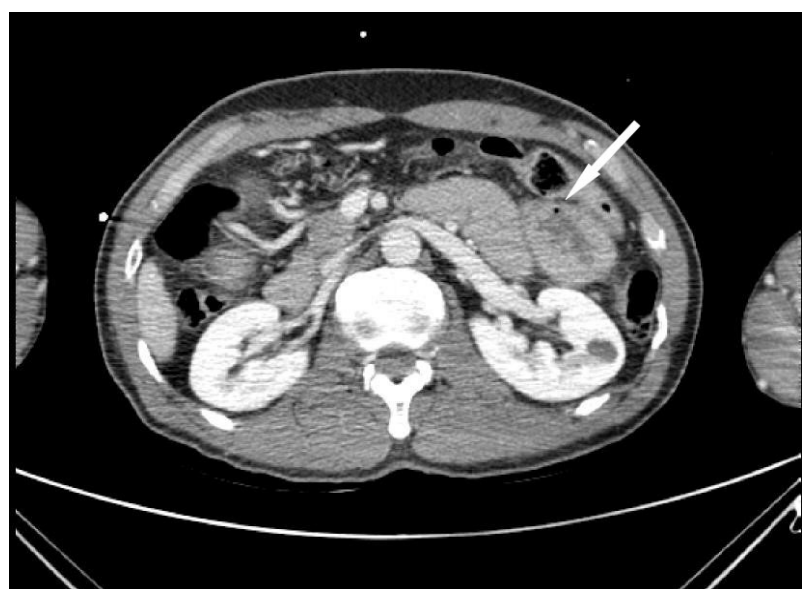

Fig. 3. Initial abdominal CT of the second patient with suspicious air bubble and edema in the jejunal wall (indicated by white arrow)

struck by a truck. Although his vital signs were stable, he was unconscious and was thus intubated in the ER. Brain CT revealed epidural hemorrhage (Fig. 2). His abdomen was soft, and no abnormal fluid or air collection was evident on the abdominal CT scan (Fig. 3). The neurosurgeons immediately brought him to the OR for craniotomy and hematoma evacuation. Post surgery, he was brought to the intensive care unit, where he developed a fever of $38^{\circ} \mathrm{C}$. After regaining consciousness, he started complaining of abdominal pain. The trauma surgeons ordered abdominal CT, which revealed a large amount of fluid collection (Fig. 4). The patient was again brought to the OR for an emergency

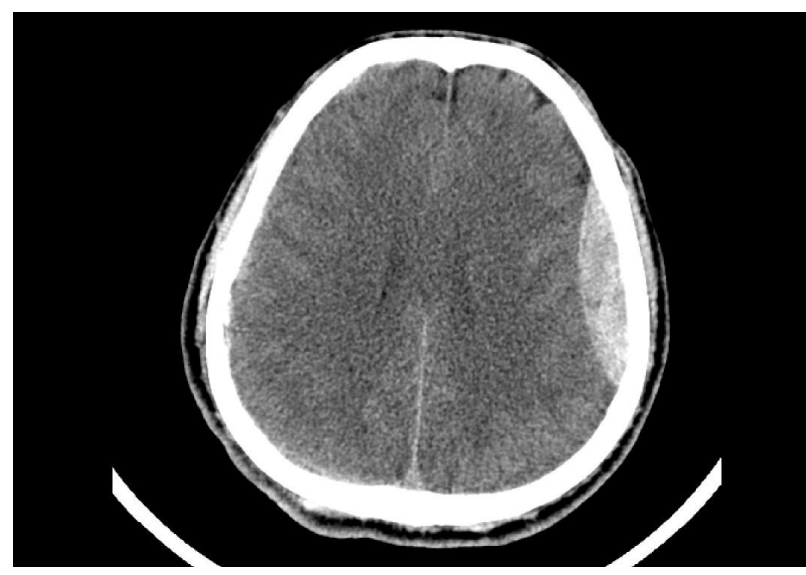

Fig. 2. Brain CT of the second patient revealing epidural hemorrhage

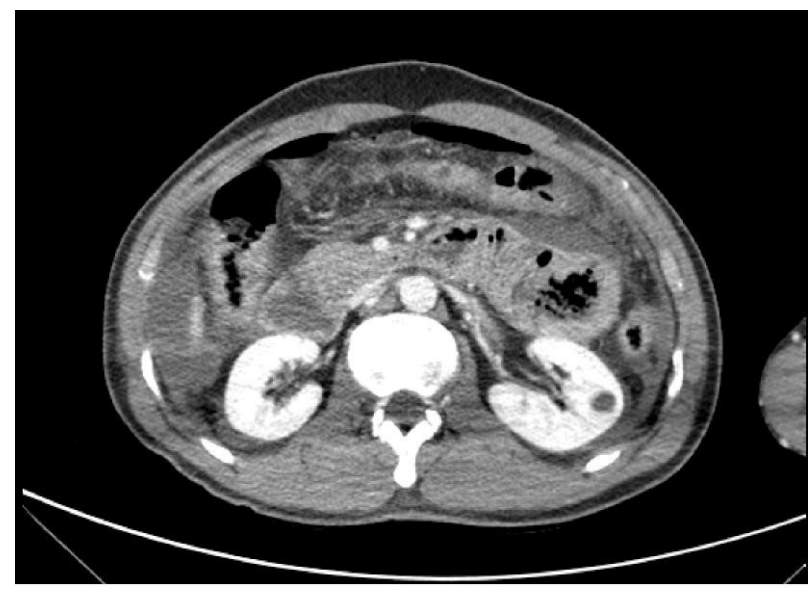

Fig. 4. Follow-up abdominal CT of the second patient revealing more edematous jejunal wall, as well as the presence of free fluid and air

laparotomy. A 3-cm perforation was found at the proximal jejunum antimesenteric border. The jejunum was mostly edematous, and a moderate amount of bowel content spillage was observed. Primary repair of the perforation was performed. The patient was discharged on postoperative day 30. Although no severe complications were observed, the patient had to be treated with 10-day intravenous antibiotics for wound infection and brain infection prevention.

\section{DISCUSSION}

Detecting bowel perforation evidences in abdominal 
CT scans was difficult in both cases. The first patient was alert and complained of severe abdominal pain. This alerted the trauma surgeons to suspect bowel injury and more thoroughly assess for evidences of bowel perforation. Even without CT scan, his typical examination results called for exploration. This was not so apparent in the second case, due to his altered mental status because his brain status required more urgent attention. However, after his mental status was resolved, he started to demonstrate signs of peritonitis; thus, bowel perforation was clearly suspected. When the patient's initial CT was reviewed, a suspicious spot of abnormal air and edema was found in the jejunal wall (Fig. 3).

Kim et al. reported that approximately $91 \%$ of CT imaging findings accurately detect gastrointestinal perforation (1). By performing CT scans and various laboratory examinations, surgeons can acquire more evidences of bowel perforation. Nevertheless, the patient's symptoms are one of the most important biomarkers. However, this may not be so apparent in cases with altered mental status. Trauma surgeons should be extra careful when diagnosing these patients.

\section{Conflict of Interest Statement}

None of authors has a conflict of interest

\section{REFERENCE}

1. Kim, H.C., Yang, D.M., Kim, S.W. et al. Gastrointestinal tract perforation: evaluation of MDCT according to perforation site and elapsed time. Eur Radiol. 2014; 24:1386 